\title{
GLOBAL FRINGE FITTING APPLIED TO MK3 VLBI DATA
}

\author{
LARS B. BÅTH \\ Onsala Space Observatory, S-439 00 Onsala, Sweden
}

\begin{abstract}
A system has been developed where data from a Mk3 VLBI processor can be read into the AIPS-package. Multiband and singleband delays can then be fitted globally by station. The technique has been used to phaserefence a background quasar to two radiogalaxies in the Abell cluster A2634.
\end{abstract}

\section{INTRODUCTION}

The Mk3 VLBI system is significantly different from the Mk2 VLBI system, and it is necessary to handle the data in a quite different way. The Mk3 system consists of a number of video converters, one for each IF-channel. These are phaselocked to the same frequency standard but otherwise independent. The seperate IF-channels are phased up by introducing a "phase-cal" signal in the form of spikes at every $\mathrm{MHz}$. These signals are used in the postprocessing stage to remove the phase differences between the IF-channels, but do not remove the phaseslope across each individual IF-band.

\section{GLOBAL FRINGE FITTING}

The data from the Mk3 processor come in the form of archivetapes, written in a backup format by the HP-computer at Haystack. A program had to be made to read and convert these data into AIPS-format. The MK3IN-program works in the following way: 1) The data are read, unpacked and converted into CONVEX format. 2) The schedule file used to run the correlator is used to select only the relevant data. Coordinates, clocks etc. are checked against this file. 3) The data are Fourier transformed from delay lags into frequency channels. The two sidebands are put together so they overlap on the central channel which is flagged since it contains the phase-calibration spike. MK3IN is run on each archive tape separately. The data are then concatenated and merged together so that only data with the highest weight (best playback) are kept.

The MK3IN program has now been rewritten into AIPS-standard and improved by W.D.Cotton of the NRAO AIPS-group and incorporated into the standard AIPS-issues. The fringe fitting program in AIPS (CALIB) had to be modified to accept larger data sets. It was also necessary to introduce several 


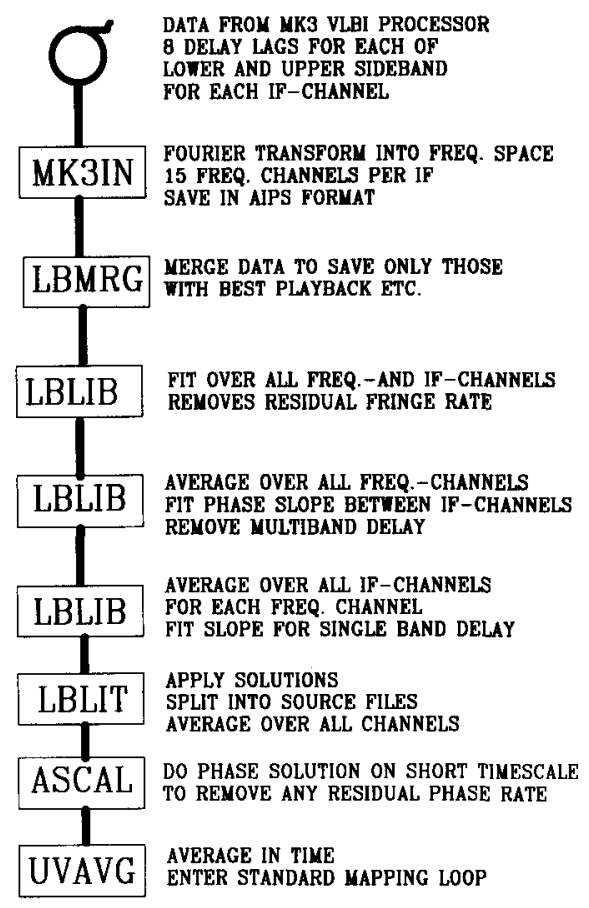

Fig. 1. Schematic representation of the technique.

new modes for it. The strategy of the technique is shown if Fig. 1. The fringe fittin of Mk3 data has to be done in three stages which are shown in Fig 2.:

1) A coarse search is done by using all IFs and frequency channels as if the data came from a Mk2 experiment with number of IFsx15 frequency channels. This step removes most of the fringe rate and also flags some "bad" solutions.

2) The multiband delays are fitted by doing a finer search after averaging each IF over all frequency channels. This can be done since the phase slope are the same over all IF channels. The data then consist of a set of frequency channels, where each IF-channel contributes to one frequency channel. This step removes the slopes between the IF-channels, i.e. the multiband delay.

3) Thereafter each frequency channel is averaged over all IF-channels and the singleband delay is fitted.

4) The data are now coherent over the frequency band and can be averaged, if so wanted, to form a data set with only one, but wide, frequency channel. Mapping and selfcalibration can then be performed as usual. More rapid phase fluctuations, due to the atmosphere etc., can now be removed by fitting station based phase offsets on a much shorter timescale. 

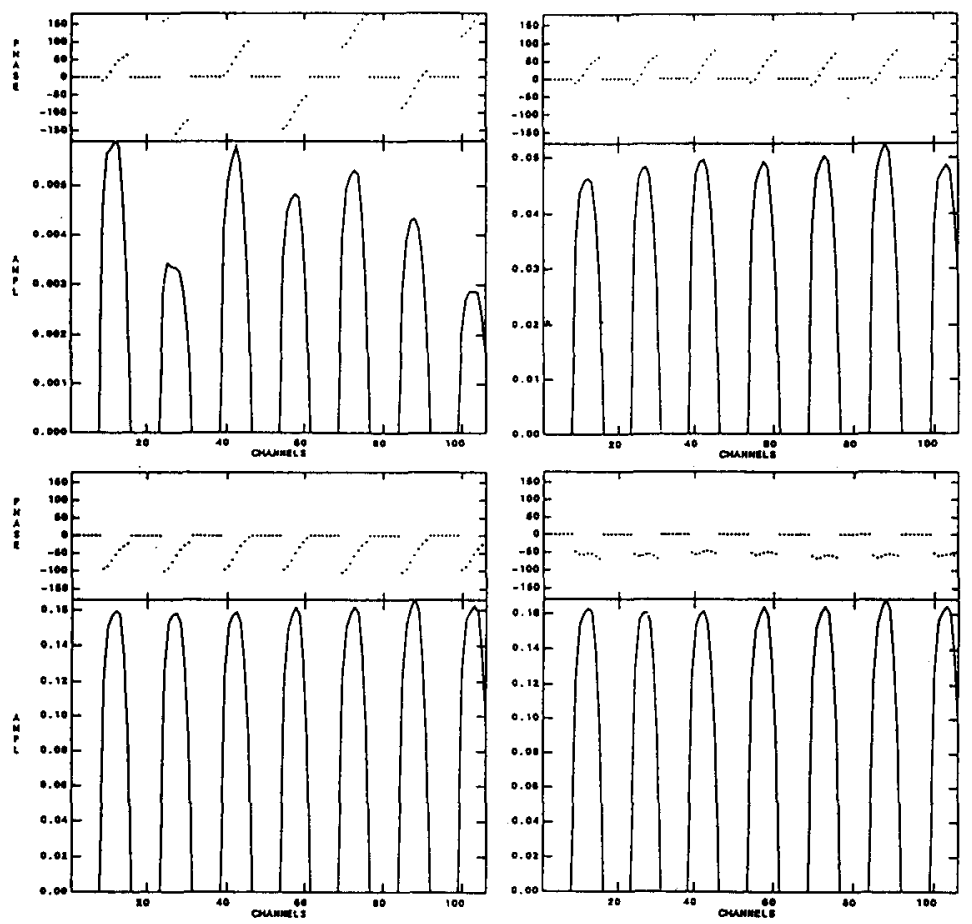

Fig. 2. The data on the baseline Haystack-Green Bank observed for $3 \mathrm{C} 84$ at $18 \mathrm{~cm}$. The panels represent the data as: original data (upper left); after rate removal (upper right); after removal of multiband delays (lower left); and after removal of single band delays (lower right)
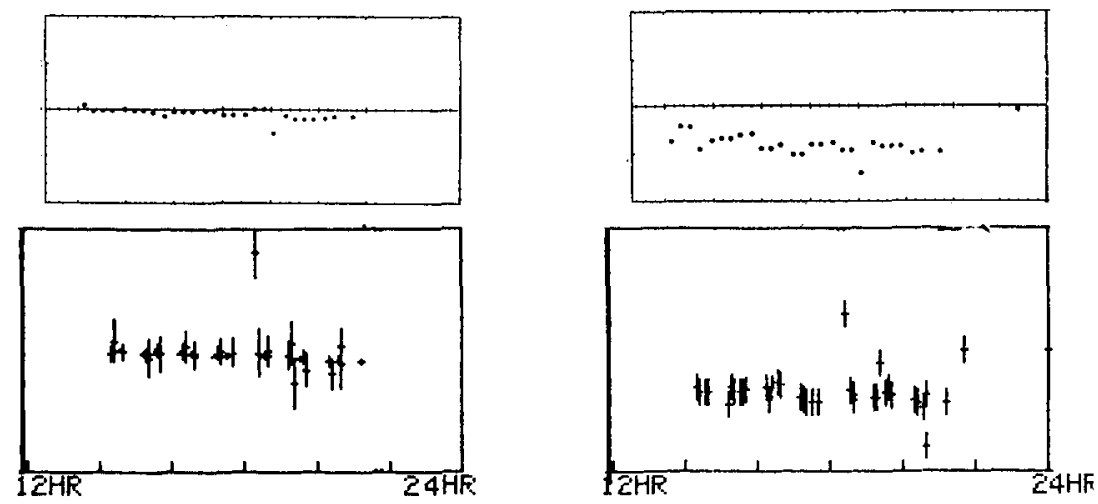

Fig. 3. Comparison of residuals from Global Fringe Fitting (upper panels) and conventional fringe fitting by baseline (lower). Left panels show residual delays $(+/-1$ microsec. $)$; right panels show residual rates $(+/-10$ picosec./sec.). 


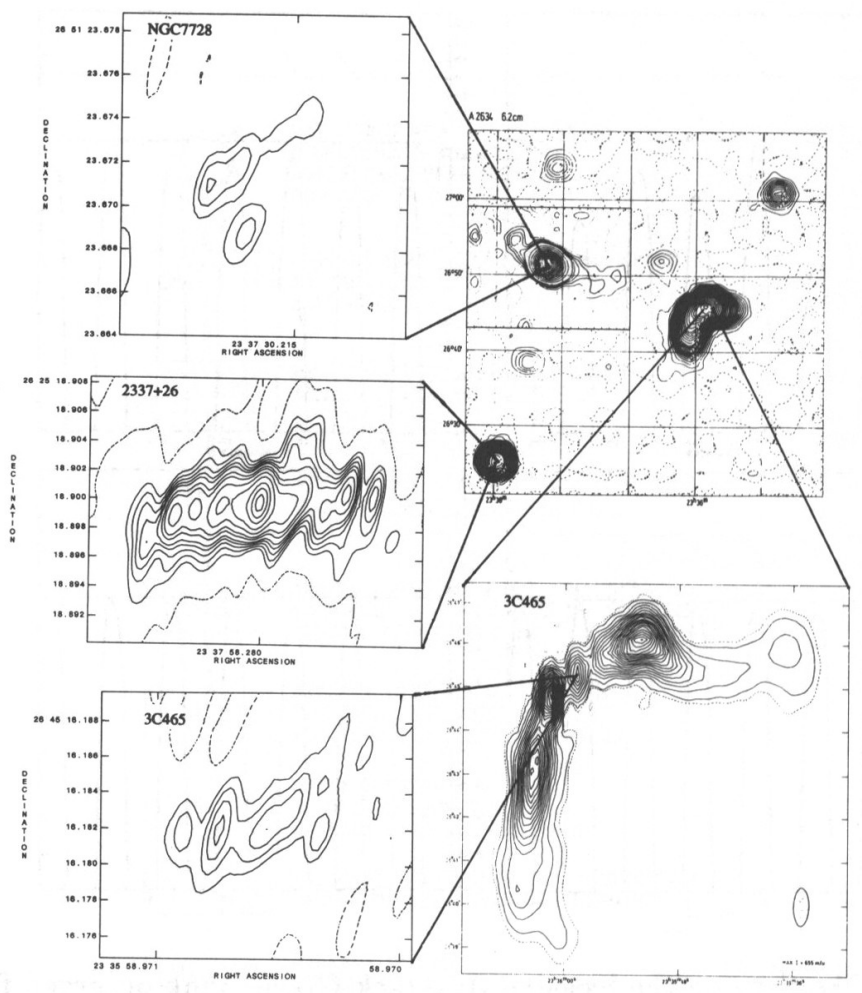

Fig. 4. Center shows a map made at Effelsberg (Wielebinski et al. 1978). Our phase referenced maps are shown on the sides. Also inserted is a Westerbork map of 3C465 (Miley and van der Laan 1973).

\section{APPLICATION TO THE ABELL CLUSTER A2634}

The above described technique was used to solve for delays and rates on the quasar $2337+26$. The solution for the telescope based residuals found on this source was then applied to the two galaxies $3 \mathrm{C} 465$ and NGC7728 in the Abell cluster A2634. The data were not averaged over frequency in this case since the positions of the sources were not sufficiently well known and the full field of view thus had to be retained. The sources were each observed every 3 mins. by switching the telescopes to each in sequence. No short-time selfcalibration of the phases was made in this case, and no selfcal was made on the two target sources 3C465 and NGC7728. Note that the VLBI source of 3C465 coincides with the position of the optical object rather than the brightest feature in the Westerbork map. The coordinates obtained by gaussian fitting to our maps are (error bars are 0".0001):

$$
\begin{aligned}
& 2337+26: \operatorname{RA}(1950)=233758.2800 \operatorname{DEC}(1950)=262518.899 \\
& 3 \mathrm{C} 465: \operatorname{RA}(1950)=233558.9707 \operatorname{DEC}(1950)=262516.181
\end{aligned}
$$


NGC7728: $\operatorname{RA}(1950)=233730.2152 \operatorname{DEC}(1950)=265123.671$

\section{REFERENCES}

Wielebinski,R., Haslam,C.G.T., Baker, J.R., and Kronberg,P.P. 1978, Astr. Ap., 67, 293

Miley,G.K. and van der Laan,H. 1973, Astr. Ap., 28, 359 